\title{
(L)-Proline catalysed efficient synthesis of 3-substituted 2,6-diarylpiperidin-4-ones
}

\author{
M. Srinivasan, S. Perumal,* and S. Selvaraj \\ Department of Organic Chemistry, School of Chemistry, Madurai Kamaraj University, \\ Madurai 625 021, India \\ E-mail: subbu_perum@rediffmail.com
}

\section{Dedicated to Professor S. Swaminathan on the occasion of his $80^{\text {th }}$ birthday}

(received 24 Dec 04; accepted 01 Feb 05; published on the web 26 Feb 05)

\begin{abstract}
(L)-Proline catalyzed Mannich reaction of ketones, aromatic aldehydes and ammonia furnishes 3-substituted 2,6-diarylpiperidin-4-ones in enhanced yields (upto five times) compared to the reaction involving ketone, aldehyde and ammonium acetate. The catalytic efficiency of proline is ascribable to the involvement of an enamine intermediate that could result from the reaction of ketones with proline and the absence of the formation of bicyclic side product in this reaction.
\end{abstract}

Keywords: Mannich reaction, (L)-proline, 2,6-diarylpiperidin-4-ones, enhanced yield, NMR

\section{Introduction}

Proline has emerged as an efficient and important enantioselective organocatalyst in several asymmetric transformations such as aldol reaction, ${ }^{1}$ conjugate addition ${ }^{2}$ involving ketones and aldehydes as nucleophilic component and additions to imines, nitroalkenes, crossed aldol reactions ${ }^{3}$ involving different aldehydes as donors and acceptors, Mannich reaction ${ }^{4}$ involving ketones, aldehydes and amines, etc. The reactivity and enantioselectivity of proline-catalyzed reactions is ascribable to a series of interactions involving proline similar to that encountered in enzyme-catalysed reactions such as substrate organization, transition state stabilization and product release. Consequently, the article by Movassaghi and Jacobsen ${ }^{5}$ has christened proline as the simplest 'enzyme'.

We have been interested in the study of synthesis, stereochemistry, biological activity and reactions of diversely substituted pipridin-4-one ring systems. ${ }^{6-9}$ The finding that (L)-proline catalyzes asymmetric Mannich reaction ${ }^{4,10}$ prompted us to examine whether the synthesis of 3substituted 2,6-diarylpiperidin-4-ones by the three-component reaction (which can be considered as a double Mannich reaction) involving ketone, aromatic aldehyde and ammonia in the presence 
of (L)-proline proceeds enantioselectively. This synthesis of piperidones was initially reported by Baliah and Noller ${ }^{11}$ from ketone, aromatic aldehyde and ammonium acetate. Although no enantioselectivity has been found in the reaction with proline under the reaction conditions employed in the present study, it is of interest to note that the yields of the 3-substituted 2,6diarylpiperidin-4-ones formed are found to be significantly higher than those reported in the literature in the presence of ammonium acetate and the results are presented in this paper. Incidentally, this work assumes importance in view of the fact that the piperidine sub-structure is widely prevalent in many natural products of biological importance.

\section{Results and Discussion}

In the present work, ethyl methyl ketone or 4-methyl-2-pentanone or ethyl acetoacetate as the ketone component was treated with substituted aromatic aldehyde and ammonia in the presence of (L)-proline to afford the respective 3-substituted 2,6-diarylpiperidin-4-ones, viz. 3-methyl-2,6diarylpiperidin-4-ones (1a-1f) or 3-isopropyl-2,6-diarylpiperidin-4-ones (1g-11) or 3ethoxycarbonyl-2,6-diarylpiperidin-4-ones (1m-1r) in 65-86\% yields (Scheme 1). All the piperidin-4-ones are obtained in the form of one diastereomer (1) consisting of racemic mixture of a pair of enantiomers. The yields and m.p. of 1a-1r are furnished in Table 1.

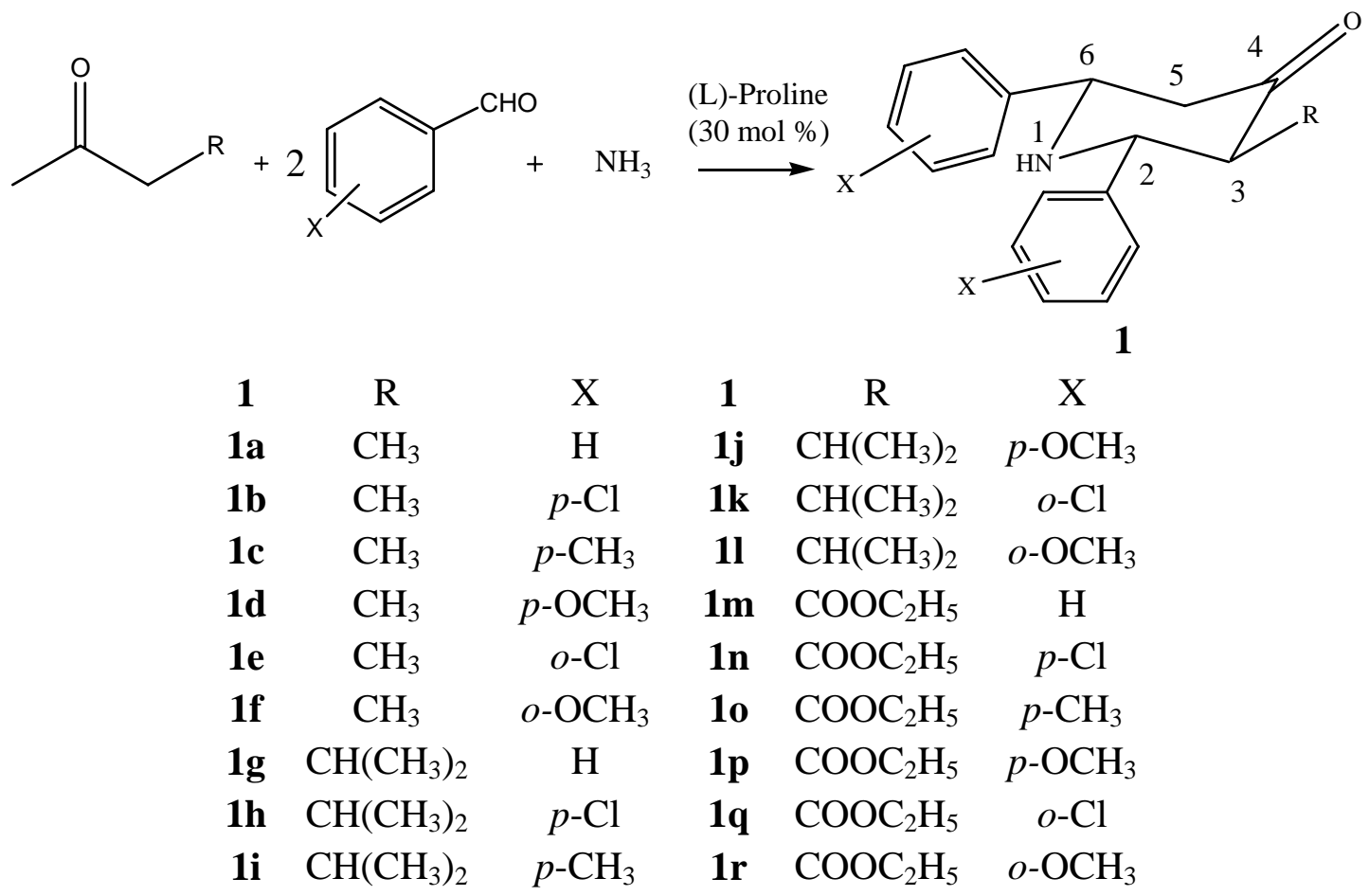

\section{Scheme 1}


Table 1. Yields and m.p. of 3-substituted 2,6-diarylpiperidin-4-ones (1a-1r)

\begin{tabular}{|c|c|c|c|c|c|c|c|c|}
\hline \multirow[t]{3}{*}{ Comp. } & \multirow{3}{*}{$\begin{array}{c}\text { m.p }{ }^{\circ} \mathrm{C} \\
\text { (Lit. } \\
\text { m.p. }{ }^{\circ} \mathrm{C} \text { ) }\end{array}$} & \multirow{3}{*}{$\begin{array}{l}\text { Lit. } \\
\text { yield } \\
(\%)\end{array}$} & \multirow{3}{*}{$\begin{array}{c}\text { Yield } \\
(\%)\end{array}$} & \multirow{3}{*}{$\begin{array}{c}\text { Yield } \\
\text { increase } \\
\text { (ratio) }\end{array}$} & \multicolumn{4}{|c|}{ Elemental Analysis } \\
\hline & & & & & \multicolumn{2}{|c|}{ Calculated (\%) } & \multicolumn{2}{|c|}{ Found (\%) } \\
\hline & & & & & $\mathrm{C}$ & $\mathrm{H}$ & $\mathrm{C}$ & $\mathrm{H}$ \\
\hline $1 \mathbf{a}$ & $86(87)^{11}$ & 40 & 75 & 1.9 & - & - & - & - \\
\hline $1 b$ & $128^{\mathrm{a}}$ & -- & 72 & -- & -- & -- & -- & -- \\
\hline 1c & $126^{\mathrm{a}}$ & -- & 68 & -- & -- & -- & -- & -- \\
\hline 1d & $137(138)^{12}$ & 49 & 66 & 1.3 & -- & -- & -- & -- \\
\hline $1 e$ & $132^{\mathrm{a}}$ & -- & 77 & -- & -- & -- & -- & -- \\
\hline 1f & $92(93)^{12}$ & 48 & 65 & 1.4 & --- & -- & -- & -- \\
\hline $1 g$ & $125(125)^{11}$ & 20 & 78 & 3.9 & -- & -- & -- & -- \\
\hline $1 \mathrm{~h}$ & 128 & -- & 75 & -- & 66.30 & 5.84 & 66.27 & 5.87 \\
\hline $1 \mathbf{i}$ & 115 & -- & 69 & -- & 82.20 & 8.47 & 82.24 & 8.51 \\
\hline $1 \mathbf{j}$ & 108 & -- & 68 & -- & 74.76 & 7.70 & 74.73 & 7.74 \\
\hline $1 \mathbf{k}$ & 121 & -- & 79 & -- & 66.30 & 5.84 & 66.34 & 5.87 \\
\hline 11 & 112 & -- & 66 & -- & 74.76 & 7.70 & 74.79 & 7.75 \\
\hline $1 \mathrm{~m}$ & $112(113)^{13}$ & $47^{\mathrm{b}}$ & 82 & 1.7 & -- & -- & -- & -- \\
\hline 1n & $128^{\mathrm{a}}$ & $14^{\mathrm{b}}$ & 74 & 5.3 & -- & -- & -- & -- \\
\hline 10 & 107 & -- & 69 & -- & 75.19 & 7.17 & 75.21 & 7.11 \\
\hline $1 p$ & $87(88)^{13}$ & $51^{\mathrm{b}}$ & 72 & 1.4 & -- & -- & -- & -- \\
\hline $1 q$ & 129 & -- & 86 & -- & 61.24 & 4.88 & 61.29 & 4.91 \\
\hline 1r & $123(124)^{13}$ & $38^{\mathrm{b}}$ & 67 & 1.8 & -- & -- & -- & -- \\
\hline
\end{tabular}

${ }^{\text {a }}$ Literature m.p. not available but ${ }^{1} \mathrm{H}$ and ${ }^{13} \mathrm{C}$ NMR spectra consistent with structure in these cases.

${ }^{\mathrm{b}}$ Yields reported as hydrochlorides.

The data in Table 1 show that an increase in yield to an extent of 1.4 to 5.3 times is found when proline is used as the catalyst. This may presumably be ascribed to the involvement of an enamine intermediate formed from the reaction of the ketone with proline in the Mannich reaction. The reaction when performed in the presence of pyrrolidine instead of proline failed to afford significant amount of piperidin-4-ones. This suggests that the carboxyl functionality of proline also presumably plays an important role in the formation of piperidin-4-ones (1).

Further, usually in the case of ammonium acetate employed as a catalyst as well as the reagent, bicyclic heterocycle (2) was also obtained as byproduct diminishing the yield of piperidin-4-ones. In contrast, the proline-catalysed reactions afforded piperidin-4-ones with no significant formation of (2). This may presumably be due to the diminished reactivity of the enamine of the initially formed piperidin-4-one (3) towards further Mannich reaction to afford (2) in view of the likely steric interactions between the aryl groups at 2- and 6- positions of the enamine of the piperidone ring and the iminium ion. 


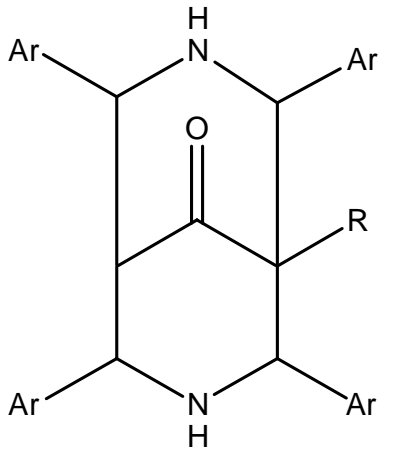

2

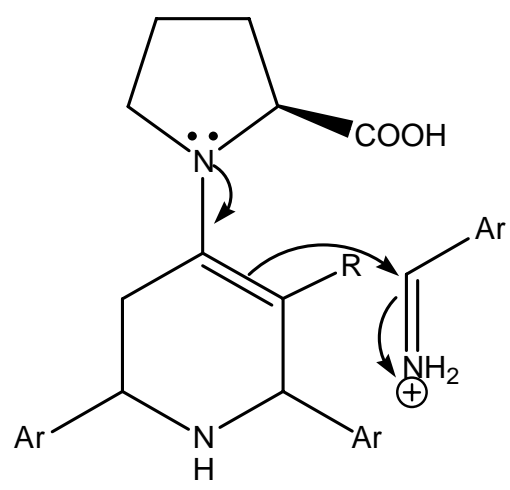

3

The ${ }^{1} \mathrm{H}$ and ${ }^{13} \mathrm{C}$ NMR spectra of 3-alkyl-2,6-diarylpiperidin-4-ones were measured and the chemical shifts assigned unambiguously employing one-dimensional ${ }^{1} \mathrm{H}$ and ${ }^{13} \mathrm{C}$ NMR and twodimensional NMR spectra such as H,H-COSY, C,H-COSY, HMBC and NOESY. The ${ }^{1} \mathrm{H}$ and ${ }^{13} \mathrm{C}$ NMR spectra of the 3-methyl-2,6-diarylpiperidin-4-ones (1a-1f), 3-isopropyl-2,6diarylpiperidin-4-ones (1g-1l) and 3-ethoxycarbonyl-2,6-diarylpiperidin-4-ones (1m-1r) are given in Table 2 and Table 3 respectively. The signals of H-2 and H-6 of the 3-substituted 2,6diarylpiperidin-4-ones are assigned on the basis of a NOESY correlation between these signals, which exist as a doublet (d) and doublet of doublets (dd) respectively.

Thus, for the representative example, $\mathbf{1 b}$ (Figure 1), the signals at $4.17 \mathrm{ppm}(\mathrm{dd})$ and 3.62 ppm (d) showing a NOESY correlation were assigned to H-6 and H-2 respectively. The other doublet at $3.76 \mathrm{ppm}$, which does not show NOESY correlation with the doublet of doublets at $4.17 \mathrm{ppm}$ could be assigned to H-3. The remaining signal, a multiplet at 2.71-2.84 ppm can be readily assigned to $\mathrm{H}-3$ and $\mathrm{H}-5$. These correlations are also supported by $\mathrm{H}, \mathrm{H}-\mathrm{COSY}$ correlations. The $J$ values for the heterocyclic ring protons disclose (i) a chair conformation for the molecule and (ii) that the aryl groups at the 2- and 6-positions and the substituent (alkyl/ester) at the 3-position are all equatorially oriented. The C,H-COSY correlations in conjunction with the unambiguous proton signal assignments of the heterocyclic ring were helpful in making firm assignment of the carbon chemical shifts of the piperidin-4-one ring system.

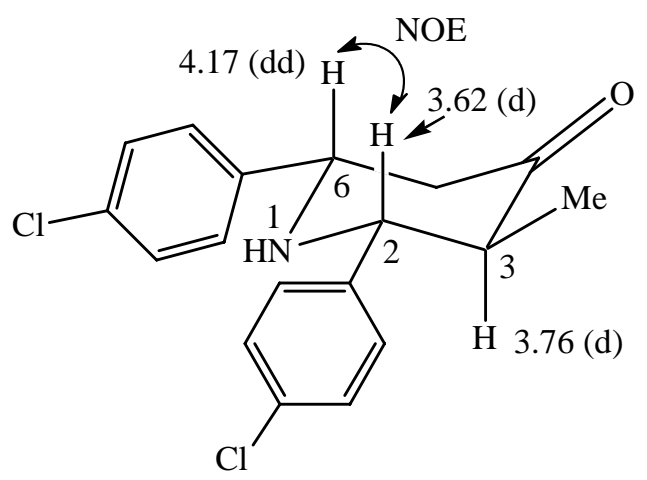

Figure 1. Two-dimensional NOESY correlation in (1b). 
Table 2. ${ }^{1} \mathrm{H}$ NMR Spectroscopic data of 3-substituted 2,6-diarylpiperidin-4-ones (1a-1r)

\begin{tabular}{|c|c|c|c|c|c|c|c|}
\hline \multirow[b]{2}{*}{ Comp } & \multicolumn{7}{|c|}{ Chemical shifts $(\delta)(J, \mathrm{~Hz})$} \\
\hline & $\mathrm{N}-\mathrm{H}$ & $\mathrm{H}-2$ & H-3 & $\begin{array}{c}\text { H-5ax, } \\
\text { eq }\end{array}$ & H-6 & $\begin{array}{l}\text { Aromatic } \\
\text { protons }\end{array}$ & Other protons \\
\hline 1a & 2.14 & $\begin{array}{c}3.63(\mathrm{~d}, \\
J=11)\end{array}$ & \multicolumn{2}{|c|}{$2.60-2.79$} & $\begin{array}{l}4.09(\mathrm{dd}, \\
J=3,11)\end{array}$ & 7.24-7.49 & $0.94\left(\mathrm{CH}_{3}\right)$ \\
\hline 1b & 2.24 & $\begin{array}{c}3.62(\mathrm{~d}, \\
J=11)\end{array}$ & $\begin{array}{l}3.76(\mathrm{~d}, \\
J=11)\end{array}$ & $\begin{array}{l}2.71- \\
2.84\end{array}$ & $\begin{array}{l}4.17(\mathrm{dd}, \\
J=4,11)\end{array}$ & $6.88-7.99$ & $0.91\left(\mathrm{CH}_{3}\right)$ \\
\hline 1c & 1.98 & $\begin{array}{c}3.68(\mathrm{~d}, \\
J=11)\end{array}$ & $\begin{array}{l}3.80(\mathrm{~d}, \\
J=11)\end{array}$ & $\begin{array}{c}2.71- \\
2.89\end{array}$ & $\begin{array}{l}4.16(\mathrm{dd}, \\
J=3,11)\end{array}$ & 6.91-7.39 & $0.97,2.22,2.25\left(\mathrm{CH}_{3}\right)$ \\
\hline 1d & 2.12 & $\begin{array}{c}3.65(\mathrm{~d}, \\
J=11)\end{array}$ & $\begin{array}{l}3.78(\mathrm{~d}, \\
J=11)\end{array}$ & $\begin{array}{c}2.70- \\
2.89\end{array}$ & $\begin{array}{l}4.15(\mathrm{dd}, \\
J=3,11)\end{array}$ & $6.68-7.29$ & $\begin{array}{c}0.93\left(\mathrm{CH}_{3}\right), 3.70,3.71 \\
\left(\mathrm{OCH}_{3}\right)\end{array}$ \\
\hline 1e & 2.03 & $\begin{array}{c}4.39(\mathrm{~d}, \\
J=11)\end{array}$ & \multicolumn{2}{|c|}{$2.52-2.83$} & $\begin{array}{l}4.58(\mathrm{dd}, \\
J=3,11)\end{array}$ & 7.18-7.89 & $0.91\left(\mathrm{CH}_{3}\right)$ \\
\hline 1f & 2.07 & $\begin{array}{c}4.51(\mathrm{~d}, \\
J=11)\end{array}$ & \multicolumn{2}{|c|}{$2.50-2.80$} & $\begin{array}{l}\text { 4.41(dd, } \\
J=3,11)\end{array}$ & $6.18-7.54$ & $\begin{array}{c}0.92\left(\mathrm{CH}_{3}\right), 3.80,3.81 \\
\left(\mathrm{OCH}_{3}\right)\end{array}$ \\
\hline $1 g$ & 1.94 & $\begin{array}{c}3.98(\mathrm{~d}, \\
J=11)\end{array}$ & \multicolumn{2}{|c|}{$2.52-2.71$} & $\begin{array}{l}\text { 4.08(dd, } \\
J=3,11)\end{array}$ & 7.24-7.49 & $1.01,1.06\left(\mathrm{CH}_{3}\right), 1.75(\mathrm{CH})$ \\
\hline 1h & 2.11 & $\begin{array}{c}4.02(\mathrm{~d}, \\
J=11)\end{array}$ & \multicolumn{2}{|c|}{$2.73-2.88$} & $\begin{array}{l}4.18(\mathrm{dd}, \\
J=4,11)\end{array}$ & $6.77-7.44$ & $0.93,1.04\left(\mathrm{CH}_{3}\right), 1.73(\mathrm{CH})$ \\
\hline $\mathbf{1 i}$ & 2.08 & $\begin{array}{c}4.06(\mathrm{~d}, \\
J=11)\end{array}$ & \multicolumn{2}{|c|}{$2.68-2.87$} & $\begin{array}{l}4.12(\mathrm{dd}, \\
J=4,11)\end{array}$ & $6.68-7.43$ & $\begin{array}{l}0.96,1.05\left(\mathrm{CH}_{3}\right), 1.77 \\
(\mathrm{CH}), 2.21,2.22\left(\mathrm{CH}_{3}\right)\end{array}$ \\
\hline $\mathbf{1 j}$ & 2.12 & $\begin{array}{l}4.11(\mathrm{~d}, \\
J=11)\end{array}$ & \multicolumn{2}{|c|}{$2.64-2.90$} & $\begin{array}{l}4.15(\mathrm{dd}, \\
J=4,11)\end{array}$ & $6.20-7.40$ & $\begin{array}{l}0.99,1.09\left(\mathrm{CH}_{3}\right), 1.74(\mathrm{CH}) \\
3.74,3.76\left(\mathrm{OCH}_{3}\right)\end{array}$ \\
\hline $1 \mathbf{k}$ & 1.93 & $\begin{array}{c}4.69(\mathrm{~d}, \\
J=11)\end{array}$ & \multicolumn{2}{|c|}{$2.51-2.73$} & $\begin{array}{l}\text { 4.61(dd, } \\
J=3,11)\end{array}$ & $7.15-7.72$ & $0.95,1.06\left(\mathrm{CH}_{3}\right), 1.73(\mathrm{CH})$ \\
\hline 11 & 1.97 & $\begin{array}{c}4.61(\mathrm{~d}, \\
J=11)\end{array}$ & \multicolumn{2}{|c|}{$2.50-2.83$} & $\begin{array}{l}\text { 4.58(dd, } \\
J=3,11)\end{array}$ & $6.20-7.40$ & $\begin{array}{c}0.96,1.07\left(\mathrm{CH}_{3}\right), 1.71(\mathrm{CH}) \\
\text { 3.64, } 3.77\left(\mathrm{OCH}_{3}\right)\end{array}$ \\
\hline $1 \mathrm{~m}$ & 2.13 & $\begin{array}{c}4.42(\mathrm{~d}, \\
J=11)\end{array}$ & $\begin{array}{l}3.68(\mathrm{~d}, \\
J=11)\end{array}$ & $\begin{array}{c}2.68- \\
2.70\end{array}$ & $\begin{array}{l}4.19(\mathrm{dd}, \\
J=4,11)\end{array}$ & $7.26-7.61$ & $0.95\left(\mathrm{CH}_{3}\right), 3.98\left(\mathrm{CH}_{2}\right)$ \\
\hline 1n & 2.14 & $\begin{array}{c}4.40(\mathrm{~d}, \\
J=11)\end{array}$ & $\begin{array}{l}3.66(\mathrm{~d}, \\
J=11)\end{array}$ & $\begin{array}{c}2.65- \\
2.69\end{array}$ & $\begin{array}{l}4.17(\mathrm{dd}, \\
J=4,11)\end{array}$ & $7.20-7.64$ & $0.95\left(\mathrm{CH}_{3}\right), 3.98\left(\mathrm{CH}_{2}\right)$ \\
\hline 10 & 2.12 & $\begin{array}{c}4.43(\mathrm{~d}, \\
J=11)\end{array}$ & $\begin{array}{c}3.67(\mathrm{~d}, \\
J=11)\end{array}$ & $\begin{array}{l}2.67- \\
2.72\end{array}$ & $\begin{array}{l}4.16(\mathrm{dd}, \\
J=4,11)\end{array}$ & $7.11-7.60$ & $\begin{array}{c}0.95\left(\mathrm{CH}_{3}\right), 2.21,2.23 \\
\left(\mathrm{CH}_{3}\right), 3.98\left(\mathrm{CH}_{2}\right)\end{array}$ \\
\hline $1 p$ & 2.13 & $\begin{array}{c}4.40(\mathrm{~d}, \\
J=11)\end{array}$ & $\begin{array}{l}3.69(\mathrm{~d}, \\
J=11)\end{array}$ & $\begin{array}{c}2.64- \\
2.70\end{array}$ & $\begin{array}{l}4.15(\mathrm{dd}, \\
J=4,11)\end{array}$ & $6.37-7.40$ & $\begin{array}{c}0.95\left(\mathrm{CH}_{3}\right), 3.71,3.74 \\
\left(\mathrm{OCH}_{3}\right), 3.98\left(\mathrm{CH}_{2}\right)\end{array}$ \\
\hline 1q & 2.01 & $\begin{array}{c}4.61(\mathrm{~d}, \\
J=11)\end{array}$ & $\begin{array}{l}3.59(\mathrm{~d}, \\
J=11)\end{array}$ & $\begin{array}{c}2.48- \\
3.10\end{array}$ & $\begin{array}{l}4.68(\mathrm{dd}, \\
J=4,11)\end{array}$ & $7.10-7.58$ & $0.81\left(\mathrm{CH}_{3}\right), 3.88\left(\mathrm{CH}_{2}\right)$ \\
\hline 1r & 2.03 & $\begin{array}{c}4.64(\mathrm{~d}, \\
J=11)\end{array}$ & $\begin{array}{c}3.57(\mathrm{~d}, \\
J=11)\end{array}$ & $\begin{array}{c}2.45- \\
3.04 \\
\end{array}$ & $\begin{array}{l}\text { 4.61(dd, } \\
J=4,12)\end{array}$ & $6.24-7.37$ & $\begin{array}{c}0.95\left(\mathrm{CH}_{3}\right), 3.55,3.87 \\
\left(\mathrm{OCH}_{3}\right), 3.98\left(\mathrm{CH}_{2}\right)\end{array}$ \\
\hline
\end{tabular}


Table 3. ${ }^{13} \mathrm{C}$ NMR Spectroscopic data of 3-substituted 2,6-diarylpiperidin-4-ones (1a-1r)

\begin{tabular}{|c|c|c|c|c|c|c|c|}
\hline \multirow{2}{*}{ Comp. } & \multicolumn{7}{|c|}{$\delta(\mathrm{ppm})$} \\
\hline & $\mathrm{C}-2$ & $\mathrm{C}-3$ & $\mathrm{C}-4$ & C-5 & C-6 & Aromatic carbons & Other carbons \\
\hline $1 \mathbf{a}$ & 68.5 & 51.0 & 209.7 & 51.5 & 61.6 & $\begin{array}{l}\text { 126.5,127.7,127.8,128.0,128.6, } \\
128.7,141.9,142.8\end{array}$ & 10.2 \\
\hline 1b & 68.3 & 51.4 & 209.5 & 51.6 & 61.9 & $\begin{array}{l}\text { 126.2,127.6,127.9,128.3,128.4, } \\
128.6,141.7,142.6\end{array}$ & 10.4 \\
\hline 1c & 68.4 & 51.2 & 209.4 & 51.7 & 61.8 & $\begin{array}{l}\text { 126.4,127.5,127.6,128.2,128.7, } \\
\text { 128.8, 141.6,142.5 }\end{array}$ & $10.3,21.1,21.3$ \\
\hline 1d & 68.1 & 51.5 & 209.5 & 51.2 & 61.1 & $\begin{array}{l}\text { 113.7,113.8,127.5,128.6,134.1, } \\
\text { 135.0,159.1,159.3 }\end{array}$ & $10.3,54.9,55.1$ \\
\hline 1e & 61.6 & 51.5 & 208.4 & 48.2 & 56.8 & $\begin{array}{l}\text { 126.5,127.3,127.5,128.2,128.5, } \\
\text { 129.3,129.5,129.7,132.3,133.6, } \\
139.2,141.9\end{array}$ & 9.7 \\
\hline 1f & 61.3 & 51.2 & 209.7 & 49.1 & 55.7 & $\begin{array}{l}\text { 110.7,111.1,121.0,121.5,126.7 } \\
128.4,130.5,132.4,156.7,157.4\end{array}$ & $10.1,54.8,55.0$ \\
\hline $1 g$ & 64.7 & 61.1 & 208.9 & 51.9 & 61.2 & $\begin{array}{l}\text { 126.5,127.7,127.9,128.0,128.6, } \\
128.7,142.1,142.8\end{array}$ & $17.6,21.0,25.9$ \\
\hline 1h & 64.3 & 61.0 & 208.7 & 51.6 & 61.4 & $\begin{array}{l}\text { 126.3,127.6,127.7,127.8,128.3, } \\
128.5,142.0,142.6\end{array}$ & $17.5,20.8,25.4$ \\
\hline $\mathbf{1 i}$ & 64.6 & 61.2 & 209.0 & 51.7 & 61.3 & $\begin{array}{l}\text { 126.4,127.8,127.9,128.1,128.4, } \\
\text { 128.6,142.2,142.9 }\end{array}$ & $\begin{array}{l}17.7,21.0,21.1 \\
21.2,25.5\end{array}$ \\
\hline $\mathbf{1 j}$ & 64.8 & 61.5 & 207.9 & 51.2 & 61.7 & $\begin{array}{l}\text { 126.7,127.5,128.2,128.3,128.6, } \\
128.8,142.6,143.4\end{array}$ & $\begin{array}{c}17.7,21.0,25.8, \\
54.7,54.9\end{array}$ \\
\hline $1 \mathbf{k}$ & 60.3 & 58.3 & 208.1 & 49.0 & 56.3 & $\begin{array}{l}\text { 127.2,127.3,128.6,129.0,129.2, } \\
\text { 129.6,129.7,132.6,133.7,139.2, } \\
139.8\end{array}$ & 18.2,21.3,26.4, \\
\hline 11 & 60.8 & 58.5 & 208.3 & 49.2 & 56.7 & $\begin{array}{l}\text { 126.9,127.7,128.4,128.5,128.8, } \\
\text { 128.9,142.8, 132.8,133.9,139.4, } \\
139.9\end{array}$ & $\begin{array}{c}17.8,21.2,26.1, \\
54.8,55.1\end{array}$ \\
\hline $1 \mathrm{~m}$ & 63.3 & 65.0 & 203.0 & 49.9 & 60.7 & $\begin{array}{l}\text { 126.4,127.5,128.0,128.3,128.5, } \\
128.7,140.3,142.0\end{array}$ & $13.9,60.8,167.9$ \\
\hline 1n & 63.1 & 64.8 & 203.2 & 49.8 & 60.5 & $\begin{array}{l}\text { 126.3,127.3,128.1,128.2,128.3, } \\
\text { 128.6,140.2,142.1 }\end{array}$ & $13.8,60.7,167.8$ \\
\hline 10 & 63.2 & 65.0 & 203.1 & 49.9 & 60.5 & $\begin{array}{l}\text { 126.2,127.4,127.8,128.1,128.2, } \\
128.6,140.1,142.0\end{array}$ & $\begin{array}{c}13.9,21.1,21.2 \\
60.8,167.5\end{array}$ \\
\hline 1p & 62.9 & 65.4 & 204.2 & 50.4 & 60.8 & $\begin{array}{l}\text { 126.4,127.2,127.6,128.3,128.5, } \\
128.6,140.0,142.1\end{array}$ & $\begin{array}{c}13.9,55.0,55.3 \\
60.8,167.7\end{array}$ \\
\hline $1 q$ & 60.4 & 58.0 & 208.2 & 48.9 & 56.8 & $\begin{array}{l}\text { 126.6,127.6,127.6,128.5,128.7, } \\
\text { 128.8,129.6,132.6,133.9,139.7, } \\
140.0,142.4\end{array}$ & $14.1,58.8,167.5$ \\
\hline 1r & 60.9 & 58.4 & 208.5 & 49.3 & 56.9 & $\begin{array}{l}\text { 126.2,127.0,127.4,128.1,128.3, } \\
\text { 128.4,129.8,132.7,134.1,139.9, } \\
140.3,142.0\end{array}$ & $\begin{array}{l}14.3,55.0,55.4, \\
58.6,167.8\end{array}$ \\
\hline
\end{tabular}




\section{Experimental Section}

General Procedures. The melting points reported in this work are uncorrected. The ${ }^{1} \mathrm{H}$ and ${ }^{13} \mathrm{C}$ NMR spectra of the 3-substituted 2,6-diarylpiperidin-4-ones (1a-1r) were measured at $300 \mathrm{MHz}$ and $75 \mathrm{MHz}$ respectively using Bruker (Avance) NMR instrument in $\mathrm{CDCl}_{3}$ and the chemical shifts referenced to tetramethylsilane. Standard Bruker software was employed to obtain both the one- and two-dimensional NMR spectra.

Synthesis of 3-substituted-2,6-diarylpiperidin-4-ones. Ammonia ( $0.4 \mathrm{ml}, 25 \%, 4.8 \mathrm{mmol})$ and (L)-proline $(0.17 \mathrm{~g}, 1.4 \mathrm{mmol})$ were dissolved in ethanol $(2 \mathrm{ml})$. Freshly distilled aromatic aldehyde $(9.6 \mathrm{mmol})$ and ketone $(4.8 \mathrm{mmol})$ were added to this solution and the mixture kept at room temperature until the mixture became a viscous liquid. Then the reaction mixture was dissolved in ether $(10 \mathrm{ml})$ and treated with aqueous hydrochloric acid [20 ml; 1:1 (v/v)]. The hydrochloride salt of the piperidin-4-one was filtered and washed with ether. The base was liberated from an alcoholic solution of the hydrochloride by adding a slight excess of aqueous ammonia and diluted with water at $0^{\circ} \mathrm{C}$. The piperidin-4-ones were crystallized from ethanol or ethanol-ethyl acetate mixture.

\section{Acknowledgements}

S.P. thanks the DST New Delhi for a major research project. Acknowledgement is also made to DST for funds under (i) IRHPA programme for the purchase of a high resolution NMR spectrometer and (ii) FIST programme and UGC for funds under the DRS and ASIST programmes.

\section{References}

1. List, B.; Lerner, R.A.; Barbas, C.F. J. Am. Chem. Soc. 2000, 122, 2395.

2. Yamaguchi, M.; Yokota, N.; Minami, T. J. Chem. Soc., Chem. Commun. 1991, 1088.

3. Peng, Y.Y.; Ding, Q.P.; Li, Z.; Wang, P.G.; Cheng, J.P. Tetrahedron Lett. 2003, 44, 3871.

4. List, B. J. Am. Chem. Soc. 2000, 122, 9336.

5. Movassaghi, M.; Jacobsen, E.N. Science 2002, 298, 1904.

6. Srinivasan, M.; Perumal, S.; Selvaraj, S. Magn. Reson. Chem. 2004, 42, 1056.

7. Srinivasan, M.; Perumal, S. unpublished results

8. Vijayabaskar, V.; Muthusubramanian, S.; Perumal, S.; Sivasubramanian, S. Indian J. Chem. 1999, 38, 909.

9. Vijayabaskar, V.; Perumal, S.; Selvaraj, S. Indian J. Chem. 1999, 38B, 771.

10. List, B.; Pojarliev, P.; Biller, W.T.; Martin, H.J. J. Am. Chem. Soc. 2002, 124, 827. 
11. Noller, C.R.; Baliah, V. J. Am. Chem. Soc. 1948, 70, 3853.

12. Baliah, V.; Gopalakrishnan, V. J. Indian Chem. Soc. 1954, 31, 250.

13. Baliah, V.; Gopalakrishnan, V.; Govindarajan, T.S. J. Indian Chem. Soc. 1954, 31, 832. 